\title{
Vertical oscillations of a coronal loop observed by TRACE
}

\author{
T. J. Wang and S. K. Solanki \\ Max-Planck Institut für Sonnensystemforschung, 37191 Katlenburg-Lindau, Germany ${ }^{\star}$ \\ Received 4 May 2004 / Accepted 26 May 2004

\begin{abstract}
We report on a loop oscillation event observed by TRACE in the $195 \AA$ bandpass at the solar limb. The difference images reveal the first evidence for vertical kink oscillations of the loop, i.e., alternately expanding and shrinking motions, in contrast to horizontal transverse loop oscillations reported before, which exhibit swaying motions. Based on the 3D geometry of the oscillating loop derived from the observation by fitting with a circular or elliptical loop model, we simulate these two kinds of global kink modes and find that only the vertical oscillations produce a signature in the difference images in agreement with the observations. We also find that the oscillating loop is associated with intensity variations. Based on the measured displacement amplitude, the simulation predicts an intensity variation of about $13 \%$ due to density changes produced by the change of the loop length. The observed intensity changes have the same sign but are considerably larger than the predictions although the error bars are also large. This suggests that these oscillations are compressible.
\end{abstract}

Key words. Sun: corona - flares - oscillations - UV radiation

\section{Introduction}

Edwin \& Roberts (1983) and Roberts et al. (1984) showed theoretically that various kinds of MHD waves and oscillations, such as slow and fast magnetoacoustic waves and torsional Alfvén waves can be supported by coronal loops. The fast modes include two solutions, termed sausage modes and kink modes, which produce oscillations that are symmetrical and asymmetrical about the axis of a hypothetical straight loop, respectively. The slow modes produce longitudinal oscillations and the torsional modes produce sheared azimuthal oscillations.

Observational confirmation of theoretical predictions obtained a big boost through the discovery of the transverse loop oscillations in TRACE data. Such oscillations have periods of the order of $5 \mathrm{~min}$ and were identified as the global kink mode (e.g. Aschwanden et al. 1999; Nakariakov et al. 1999). Later, hot loop oscillations in Doppler shifts with periods on the order of 20 min were detected by the SUMER spectrometer onboard $\mathrm{SOHO}$, and interpreted in terms of the standing slow mode (e.g. Wang et al. 2002, 2003; Ofman \& Wang 2002). Also, propagating waves have been detected in coronal loops by SOHO/EIT and TRACE as intensity variations (e.g. Berghmans \& Clette 1999; De Moortel et al. 2000), and identified as slow mode waves (Nakariakov et al. 2000). The observations of such oscillations and waves are leading to a diagnostic tool, dubbed coronal seismology, for determining unknown coronal parameters, such as the magnetic field in coronal loops (e.g. Roberts 2000; Nakariakov \& Ofman 2001).

Send offprint requests to: T. J. Wang,

e-mail: wangtj@linmpi.mpg.de

^ Previously known as MPI für Aeronomie.
For a straight, gravity-free cylindrical model of a magnetic loop, there is no distinction, beyond the purely geometric, between kink modes that perturb the cylinder in different directions. In reality, however, magnetic loops are bent, as their name implies, with their footpoints anchored in the photosphere. Then we need to distinguish between two kinds of global kink oscillations (Edwin \& Roberts 1983), one with swaying motions perpendicular to the loop plane (termed in the following horizontal oscillations), the other with motions polarized in the loop plane. Two types of such oscillations are possible. The first, termed global vertical oscillations, causes the whole loop to expand and shrink while conserving its shape. The second distorts the loop while conserving its length (we call them distortion oscillations). The previous observational studies of kink oscillations usually considered on-disk oscillation events seen by TRACE, which indicate the presence of horizontal loop oscillations by clear variations of the inclination of loops. In this study, we report the first observational evidence for the vertical loop oscillation.

\section{Observations}

A loop oscillation event on 17 April 2002 was detected by TRACE at the northwest limb (see Fig. 1). The observations were made with the $195 \AA$ bandpass, at a full resolution of $0.5 \operatorname{arcsec}$ pixel $^{-1}$ and with a cadence of $17 \mathrm{~s}$. The main lines in the employed bandpass are the 1.5 MK Fe XII line and the 20 MK Fe xxIv line. Although this TRACE oscillating loop was also situated in the field of view of the SUMER slit and simultaneous spectra were recorded in the $\mathrm{S}_{\text {III, }} \mathrm{Cax}$ and Fe xix lines with a $50 \mathrm{~s}$ cadence, no signatures of the oscillation are found in these lines, possibly because the loop is relatively 


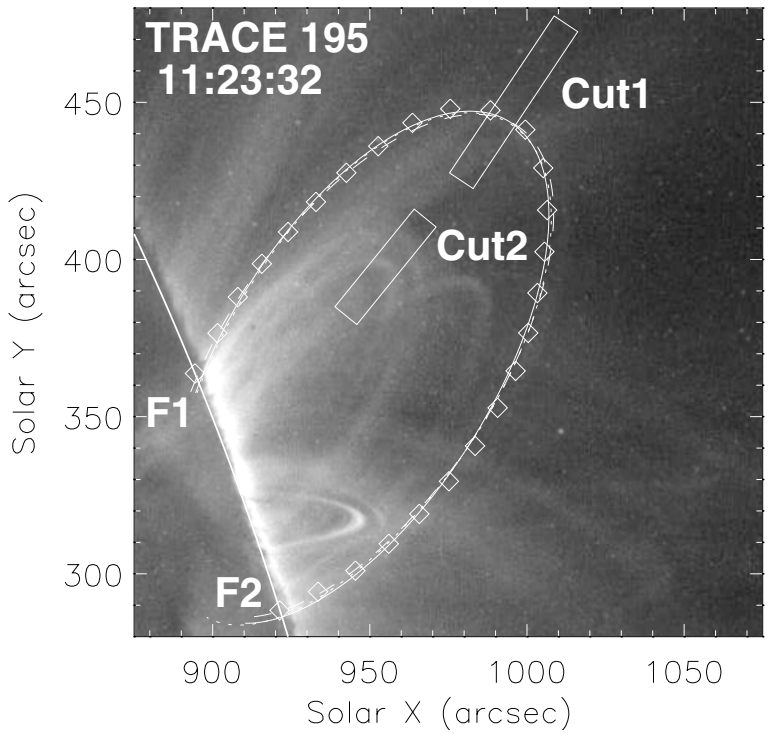

Fig. 1. A TRACE image containing the analyzed coronal loop (outlined by the diamonds), which is fit assuming three loop geometries: circular loop (solid curve), fat (dotted curve) and thin (dashed curve) elliptical loops. Both footpoints of the loop are located behind the solar limb. The boxes mark the locations of two cuts shown in Fig. 2.

cool and thus not seen in Fe XIx, while Cax is rather insensitive to loop oscillations, since it generally suffers from a strong background contribution. Thus we only focus on the analysis of TRACE data for this event.

\section{Results}

The oscillating loop is only weakly visible in the TRACE images. The loop is outlined in Fig. 1 just before it starts to oscillate. This loop first appeared during the period 10:30-10:40 UT, and was most prominently seen around 10:50 UT (see Fig. 3a). Both footpoints of this loop were located behind the limb. SOHO/EIT images from previous days show that this loop belonged to AR 9893, where most loop systems were oriented clockwise relative to the solar equator. This implies that the northern footpoint (F1) is nearer to the observer, in agreement with the stronger brightness at F1 than at F2. We determined the geometrical parameters of the loop, by using a method similar to that employed by Aschwanden et al. (2002), but keeping the positions of both footpoints as free parameters of the fit. We carried out different fits, assuming the loop to be circular or elliptical. For the elliptical loop model, two solutions were found, corresponding to the cases when the major axis is parallel (i.e. a fat loop) or perpendicular (i.e. a thin loop) to the loop footpoint baseline. The parameters for the three loop shapes are listed in Table 1. For the loop length we obtain $L \approx 300-400 \mathrm{Mm}$, for the azimuth angle of the loop baseline to the EW direction $\alpha \approx 30-50^{\circ}$, and for the inclination angle of the loop plane to the vertical $\theta \approx 20-40^{\circ}$. The loop is inclined northward, i.e. away from the observer.

Figure $2 \mathrm{a}$ shows the intensity evolution along a slice at the apex of the oscillating loop (marked Cut1 in Fig. 1). The oscillation event started at about 11:24 UT. The whole loop system first moved outward with an initial velocity of

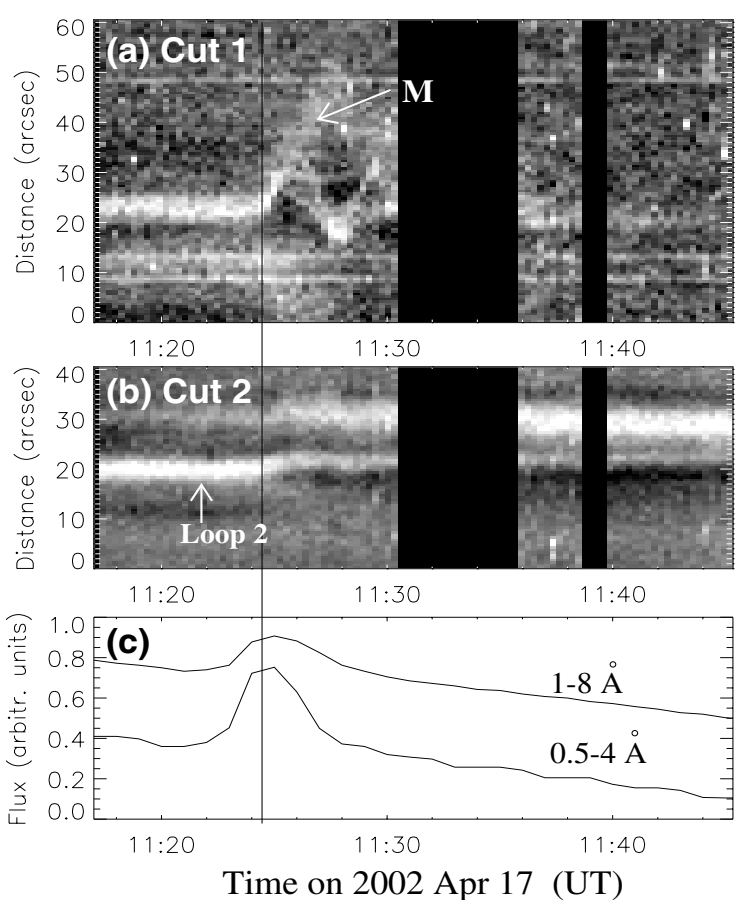

Fig. 2. Time slices of TRACE intensity along a) Cut1 and b) Cut2, whose location is marked in Fig. 1, where the intensity is integrated along its narrower side of a width of 9 arcsec. In both a) and b), the background intensity has been removed in order to enhance clarity, and the black strips indicate data gaps. c) Evolution of GOES X-ray flux in two bandpasses.

$V_{\text {osc }} \approx 130 \mathrm{~km} \mathrm{~s}^{-1}$, measured along the slice. Then part of the loop system moved back and forth to show oscillations, while another, fainter part continued to move outward with a speed of about $90 \mathrm{~km} \mathrm{~s}^{-1}$ (marked M in Fig. 2a). The intensity of the oscillating loop decreased as it moved outward and increased again when it moved back towards the solar limb. An intensity peak is clearly seen when the loop is at the lowest position. Estimates show that this is not an artifact of the longer integration of the nearly stationary loop at that time. The oscillation is visible for one and a half periods before a data gap of 6 min interrupts the sequence. By fitting the oscillation with a damped sine function (following the method described by Nakariakov et al. 1999), we obtain a displacement amplitude of $A=7900 \mathrm{~km}$, an oscillation period of $P=3.9 \mathrm{~min}$, and a decay time of $T_{\mathrm{d}}=11.9 \mathrm{~min}$. For comparison, the intensity evolution for another slice at an inner loop system (marked Cut 2 in Fig. 1) is shown in Fig. 2b. We find that a loop (marked Loop2 in Fig. 2b) got shifted upward and possibly partially erupted as suggested by the dimming. Clearly, Loop2 gets disturbed and the loop oscillation starts when the GOES X-ray flux shown in Fig. 2c reaches its peak (marked with a vertical line in all frames). This suggests that the loop oscillation is probably triggered by a blast shock produced in a strong flare occurring behind the limb.

Figure 3 shows difference images, the difference between two images taken well before the start of the oscillation being plotted in Fig. 3a. The loop that later oscillated is visible in this image, since it gradually brightened prior to the oscillation. In Figs. 3b-d we plot running differences between images 
Table 1. Geometrical parameters of the oscillating loop obtained for three loop geometries ${ }^{a}$.

\begin{tabular}{lccccccccc}
\hline \hline Models & $\begin{array}{c}l_{1} \\
(\mathrm{deg})\end{array}$ & $\begin{array}{c}b_{1} \\
(\mathrm{deg})\end{array}$ & $\begin{array}{c}l_{2} \\
(\mathrm{deg})\end{array}$ & $\begin{array}{c}b_{2} \\
(\mathrm{deg})\end{array}$ & $\begin{array}{c}\alpha \\
(\mathrm{deg})\end{array}$ & $\begin{array}{c}\theta \\
(\mathrm{deg})\end{array}$ & $\begin{array}{c}h_{0} \\
(\mathrm{Mm})\end{array}$ & $\begin{array}{l}a \text { and } b(\text { or } r) \\
(\mathrm{Mm})\end{array}$ & $\begin{array}{c}L \\
(\mathrm{Mm})\end{array}$ \\
\hline Circle & 93.5 & 22.0 & 99.5 & 17.0 & 39.8 & 30.5 & 48 & $r=66$ & 312 \\
Fat ellipse & 94.5 & 22.0 & 104.0 & 17.0 & 27.8 & 22.0 & 48 & $a=104$ and $b=60$ & 391 \\
Thin ellipse & 94.0 & 22.0 & 97.5 & 17.2 & 53.9 & 36.5 & 51 & $a=49$ and $b=73$ & 300 \\
\hline
\end{tabular}

${ }^{a}\left(l_{1}, b_{1}\right)$ and $\left(l_{2}, b_{2}\right)$ are the heliographic longitude and latitude relative to Sun center of the two footpoints of the loop. $\alpha$ is the azimuth angle of the loop baseline to the east-west direction. $\theta$ is the inclination angle of the loop plane to the vertical. $h_{0}$ is the height of the ellipse's (or circle's) center measured in the loop plane. $a$ and $b$ are half lengths of the elliptical axes lying parallel and perpendicular to the loop footpoint baseline, respectively; $r$ is the radius of the circular loop. $L$ is the length of the loop.

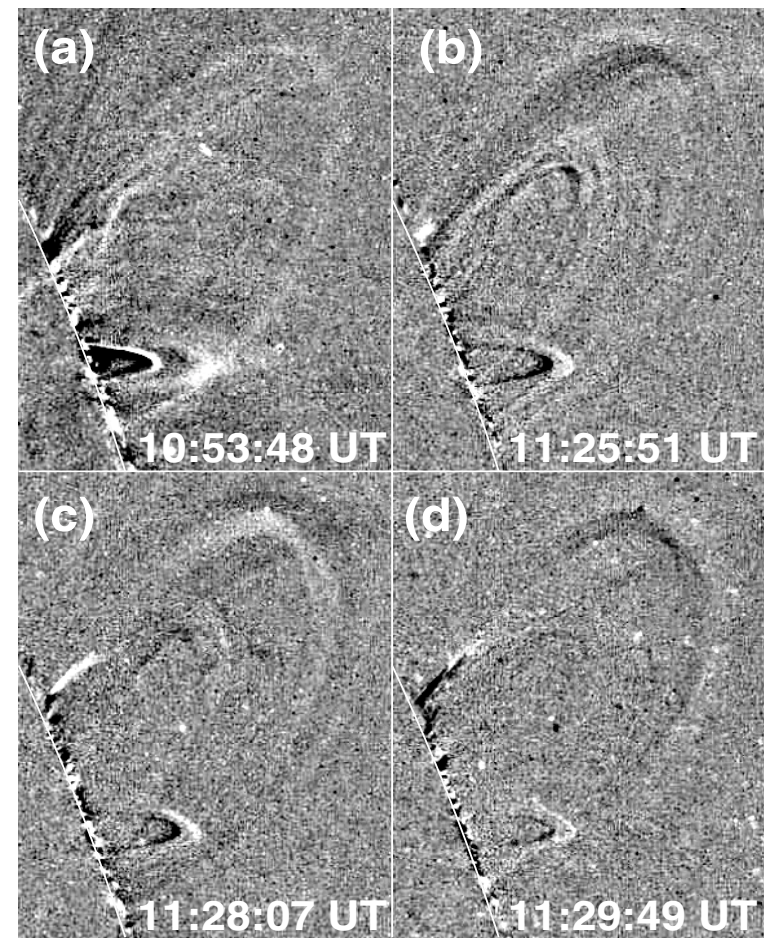

Fig. 3. Observations of the oscillating TRACE loop on 17 April 2002. a) A difference image before the event, which is produced by subtracting the image at 10:35:06 from one at 10:53:48. b)-d) Running difference images with an interval of $\sim 2$ min. Black indicates where the loop was in the earlier image and white where it has moved to.

recorded about 2 min apart during the oscillation, corresponding to roughly half a wave period, where the white loop indicates the position at the time marked in the images, while the dark loop marks the position in the earlier image. We see that the white loop is located either outside (Figs. $3 \mathrm{~b}$ and d) or inside (Fig. 3c) the dark loop and that the most obvious displacements happen near and slightly northward of the loop top.

In order to better identify the observed oscillation mode we have computed the signatures of horizontal and vertical oscillations in a synthetic loop. In this simulation, we assume that the loop width is $10^{\prime \prime}$ and the brightness exhibits a Gaussian distribution across the loop. The radial displacements along the loop follow $\Delta R(\phi)=0.03 R(\phi) \sin \phi\left(0^{\circ} \leq \phi \leq 180^{\circ}\right)$, where $R(\phi)$ is the distance from the midpoint of the loop footpoint baseline to a point on the loop, which has an azimuth angle of $\phi$ relative
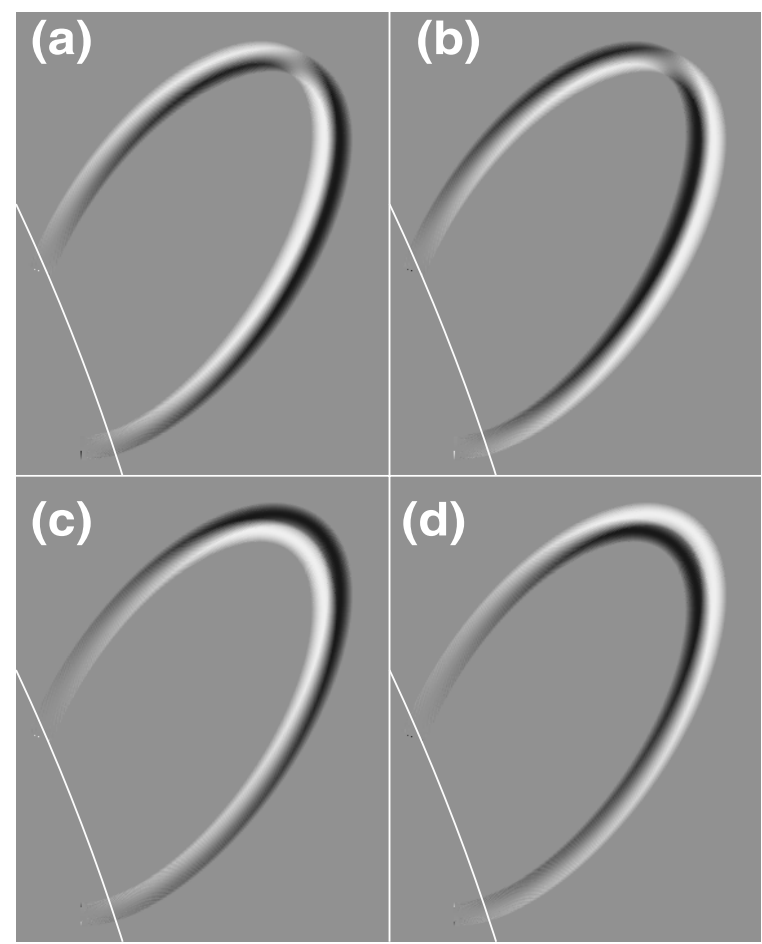

Fig. 4. Difference images of simulated loop oscillations, with a geometry derived from the observation based on a circular loop model (see Table 1). a)-b) Horizontal oscillations. c)-d) Vertical oscillations.

to the loop baseline. For the simulated horizontal oscillations, we assume that a rigid loop deviates away from its equilibrium position by rotating by an angle of $\Delta \theta=1.5^{\circ}$ relative to the loop baseline (which is taken to be the axis of rotation). The remaining loop parameters are taken from Table 1. A simulation is run for each of the three geometries deduced from the observations.

The results of the simulation are shown in Fig. 4 as difference images between times of successive maximum displacement of the loop. Horizontal and vertical oscillations exhibit distinctly different signatures. Whereas the vertical oscillation appears as a uniform displacement (Figs. 4c and d), with a maximum contrast near the loop-top, the horizontal oscillation exhibits a cross-over between the black and the white shading close to that location (Figs. $4 \mathrm{a}$ and b). A comparison with Fig. 3 shows that the computed signature of the vertical oscillation is reasonably consistent with the observations, while the 
horizontal oscillation clearly contradicts them. Note that the rigid loop assumption adopted in the simulation of horizontal oscillations may not be quite correct, because in reality the legs of the loop are expected to sway with a smaller angular amplitude than the top due to the influence of magnetic curvature forces, which resist the sharp bending of field lines. However, this approximation should not change the main characteristics that distinguish the horizontal oscillation from the vertical oscillation. The simulations shown in Fig. 4 are based on the circular loop model, but very similar results are obtained for the two elliptical loop geometries listed in Table 1.

\section{Discussion}

A number of transverse loop oscillations have been reported in a statistical study by Aschwanden et al. (2002), and most of them manifest clearly horizontal motions. The event analyzed here, however, for the first time reveals evidence of vertical oscillations of a TRACE loop. Horizontal and vertical oscillations are two different solutions of the kink modes in a curved, linetied loop. The former produces little change in the loop length, and therefore, shows nearly incompressible, Alfvénic properties. The latter is associated with expanding and shrinking motion, so that if the gas content and the cross-section of the loop remain unchanged over a wave period, this mode should lead to compressions and rarefactions of the gas, i.e. to intensity perturbations. Note that this compressibility is introduced by the nonlinearity connected with the effects of finite amplitude, but is not a property of the mode itself.

According to Fig. 2 the oscillating loop exhibited brightness variations, especially a clear intensity enhancement when the loop shrank. Assuming a circular loop geometry we measure the maximum displacement of the loop at Cut1 to be $\Delta R \approx 7.9 \mathrm{Mm}$ and its radial distance from the midpoint of the loop baseline to be $R=112 \mathrm{Mm}$. We also calculate that the angle between this radial vector and Cut 1 is about $8^{\circ}$, so that the $\Delta R$ measured in Cut 1 should correspond very closely to the true amplitude. We obtain a relative displacement amplitude of $\Delta R / R \approx 7.1 \%$. Assuming this value to be the maximum amplitude along the loop and also assuming the displacement along the loop to follow a sine curve, we obtain the oscillation amplitude of the loop length to be $\Delta L / L \approx 6.6 \%$. If the loop cross-section does not change and the possibility of fieldaligned flows at the footpoints is neglected during the oscillations, we estimate the intensity variation according to mass conservation in the loop as,

$\frac{\Delta I}{I} \sim \frac{2 \Delta n}{n} \sim \frac{2 \Delta L}{L}=0.13$

The $\Delta I / I$ of the oscillating loop that we measure is much larger. It turns out to be $-0.9 \pm 0.5$ for the peak position and $1.1 \pm 1.0$ for the valley position in Fig. 2a, where the intensity variations are relative to the intensity at an intermediate phase of the oscillation (i.e., the middle of the peak and valley positions) and the background intensity has been removed. In spite of the large errors, the intensity variations do appear to be much larger than expected without any modulations of the loop's cross-section.

In a $2 \mathrm{D}$ and zero- $\beta$ plasma slab model of a prominence fibril, the theory worked out by Joarder et al. (1997) has predicted the existence of two global oscillation modes, called an Alfvén string mode and a string-type magnetic Love (fast body kink) mode, which are similar to the horizontal oscillation and vertical oscillation in a coronal loop discussed here. They show that the string-type magnetic Love modes are highly compressible and have phase speeds higher than the Alfvén string modes, thus producing shorter periods than the latter. In our study, the vertical oscillation has a period of $P=234$ s for a loop length of $L \approx 300-400 \mathrm{Mm}$. This value is indeed shorter than periods of the horizontal oscillations for a similar loop length. For 6 such cases (2a, 3a, 4a, 10a, 11a, and 14a) measured in Aschwanden et al. (2002), we find that $P=286-522 \mathrm{~s}$ with a mean value of $389 \pm 82 \mathrm{~s}$ and $L=256-406 \mathrm{Mm}$ with a mean value of $311 \pm 63 \mathrm{Mm}$.

The theory of the oscillation modes of a curved, line-tied magnetic cylinder model of a coronal loop needs to be developed in order to understand the observations of $\Delta I / I$ and to allow a more realistic application of coronal seismology. Note, however, that the source of the large $\Delta I / I$ could also lie in the inhomogeneous background brightness distribution, which makes interpretation uncertain. It is therefore important to detect further cases of vertical oscillations for which the background emission is weak compared to the loop emission.

Acknowledgements. We would like to thank the anonymous referee for his valuable comments in improving the manuscript.

\section{References}

Aschwanden, M. J., Fletcher, L., Schrijver, C. J., \& Alexander, D. 1999, ApJ, 520, 880

Aschwanden, M. J., De Pontieu, B., Schrijver, C. J., \& Title, A. 2002, Sol. Phys., 206, 99

Berghmans, D., \& Clette, F. 1999, Sol. Phys., 186, 207

De Moortel, I., Ireland, J., \& Walsh, R. W. 2000, A\&A, 355, L23

Edwin, P. M., \& Roberts, B. 1983, Sol. Phys., 88, 179

Joarder, P. S., Nakariakov, V. M., \& Roberts, B. 1997, Sol. Phys., 173, 81

Nakariakov, V. M., Ofman, L., DeLuca, E. E., et al. 1999, Science, 285,862

Nakariakov, V. M., Verwichte, E., Berghmans, D., \& Robbrecht, E. 2000, A\&A, 362, 1151

Nakariakov, V. M., \& Ofman, L. 2001, A\&A, 372, L53

Ofman, L., \& Wang, T. J. 2002, ApJ, 580, L85

Roberts, B., Edwin, P. M., \& Benz, A. O. 1984, ApJ, 279, 857

Roberts, B. 2000, Sol. Phys., 193, 139

Wang, T. J., Solanki, S. K., Curdt, W., Innes, D. E., \& Dammasch, I. E. 2002, ApJ, 574, L101

Wang, T. J., Solanki, S. K., Innes, D. E., Curdt, W., \& Marsch, E. 2003, A\&A, 402, L17 